\title{
5-Aminolevulinic acid combined with ferrous iron improves glucose tolerance in high-fat diet-fed mice via upregulation of glucose transporter 1
}

\author{
YASUSHI KURODA ${ }^{1}$, ATSUKO KAMIYA ${ }^{1}$, TAKUYA ISHII ${ }^{1}$, MASAHIRO ISHIZUKA ${ }^{1}$, \\ YOKO YAMASHITA $^{2}$ and HITOSHI ASHIDA ${ }^{2}$ \\ ${ }^{1}$ SBI Pharmaceuticals Co., Ltd., Tokyo 106-6020; ${ }^{2}$ Department of Agrobioscience, \\ Graduate School of Agricultural Science, Kobe University, Kobe, Hyogo 657-8501, Japan
}

Received March 25, 2021; Accepted September 16, 2021

DOI: $10.3892 /$ etm.2021.10889

\begin{abstract}
Decreased mitochondrial metabolism suppresses glucose metabolism, resulting in obesity and diabetes. The present study aimed to investigate mechanisms underlying the 5-aminolevulinic acid (5-ALA) hydrochloride-mediated increase in glucose uptake in high-fat diet (HFD)-fed mice in vivo and $\mathrm{C} 2 \mathrm{C} 12$ myotube cells in vitro. $\mathrm{C} 57 \mathrm{BL} / 6 \mathrm{~N}$ male mice (20 weeks old) were fed either HFD or normal diet (ND) for 4 weeks. A total of five HFD-fed mice were orally administered with $300 \mathrm{mg} / \mathrm{kg}$ 5-ALA hydrochloride and $47.1 \mathrm{mg} / \mathrm{kg}$ sodium ferrous citrate (SFC; HFD + 5-ALA/SFC), whereas ND and other HFD-fed mice were orally administered with saline. After 4 weeks, these mice were intraperitoneally administered with $2 \mathrm{~g} / \mathrm{kg}$ glucose and $3.2 \mathrm{mg} / \mathrm{kg}$ 2-deoxyglucose (2DG) for intraperitoneal glucose tolerance test (IPGTT) and glucose uptake test. Body weights, plasma glucose levels and the area under the curve of IPGTT were lower in mice treated with HFD + 5-ALA/SFC compared with in those treated with HFD alone. 2DG uptake in the gastrocnemius muscle and heart were more significantly improved in the HFD + 5-ALA/SFC mice compared with the HFD-fed mice. Furthermore, 5-ALA/SFC increased 2DG uptake in $\mathrm{C} 2 \mathrm{C} 12$ cells to a similar level to the insulin-treated group. Moreover, it increased glucose transport (GLUT)1 translocation in the plasma membrane by 2.5 -fold relative to the controls without affecting GLUT1 expression; however, it had no effect on GLUT4 translocation. Therefore,
\end{abstract}

Correspondence to: Dr Atsuko Kamiya, SBI Pharmaceuticals Co., Ltd., Izumi Garden Tower 20F, 1-6-1 Roppongi, Minato-ku, Tokyo 106-6020, Japan

E-mail: atsuzuki@sbigroup.co.jp

Abbreviations: 5-ALA, 5-aminolevulinic acid; SFC, sodium ferrous citrate; HFD, high-fat diet; ND, normal diet; IPGTT, intraperitoneal glucose tolerance test; 2DG, 2-deoxyglucose

Key words: high-fat diet, glucose tolerance, 5-aminolevulinic acid, muscle tissue, mitochondrial function
5-ALA/SFC enhanced gastrocnemius and cardiac glucose uptake in HFD-fed mice, and upregulated GLUT1 translocation to the plasma membrane, but not GLUT4 in C2C12 myotube cells. Therefore, it could potentially be used as a novel drug for the treatment of diabetes.

\section{Introduction}

The endogenous amino acid 5-aminolevulinic acid (5-ALA) is widely distributed in both animals and plants and is synthesized by glycine condensation and succinyl-CoA via the mitochondrial ALA synthase in animal cells (1). It is then further converted into protoporphyrin IX (PPIX) due to well-known subsequent enzymatic reactions (1). Next, a divalent iron is coordinated to PPIX for heme synthesis (1). A synthesized heme binds to corresponding apoproteins and becomes a hemeprotein (2).

Furthermore, 5-ALA hydrochloride has been orally administered for the photodynamic diagnosis using specific accumulation of fluorescent PPIX in tumor tissues of patients with cancer (3-5). Conversely, 5-ALA is metabolized to heme combined with iron in normal tissues, producing hemeproteins that exhibit several biological effects such as oxidoreductive activities (catalase and oxidase), electron transport (cytochrome c), and oxygen delivery (myoglobin and hemoglobin) (6,7). Externally administered 5-ALA significantly affects energy metabolism of the whole body through mitochondrial hemeproteins synthesized from 5-ALA, which is reduced with aging (8). Ingested 5-ALA is reported to enhance energy metabolism by activating the mitochondrial electron transport system that promotes ATP synthesis in the mouse liver (9). Furthermore, 5-ALA with sodium ferrous citrate (SFC) is reported to augment exercise efficiency in elderly women (10). Our previous studies reported that 5-ALA with SFC exerts anti-diabetic effects in Zucker diabetic fatty $(\mathrm{ZDF})$ rats and patients with borderline diabetes $(11,12)$. Orally administered 5-ALA/SFC reduces fasting blood glucose and hemoglobin A1c levels and improves glucose tolerance in ZDF rats without affecting insulin secretion (11). The lipid content in 3T3-L1 adipocytes is decreased by 5-ALA/SFC and glucose uptake in 3T3-L1 and L6 myotube cells is induced (13). 
Glucose uptake from blood to cells is caused by insulin secreted from the pancreas, which translocates glucose transporter (GLUT) 4 from the cytoplasm to the plasma membrane in muscles and adipose tissues (14,15). Overall, 14 GLUT isoforms have been detected, and their expression levels differ among organs (16). Because the administration of 5-ALA/SFC decreases blood glucose level in vivo and enhances glucose uptake in vitro, some glucose transporters in tissues are assumed to be activated; however, no study has identified the involved tissues and transporters.

Therefore, the present study analyzed glucose kinetics in vivo and the expression level of GLUT isoforms in the plasma membrane to identify a glucose transporter activated by 5-ALA/SFC. 5-ALA/SFC was administered to high-fat diet (HFD)-fed mice to elucidate the target tissues that reduced the blood glucose level. Furthermore, the effect of 5-ALA/SFC on glucose uptake in $\mathrm{C} 2 \mathrm{C} 12$ myotube cells was examined, and the expression level and type of glucose transporters in the plasma membranes of $\mathrm{C} 2 \mathrm{C} 12$ cells were investigated.

\section{Materials and methods}

Reagents. 5-ALA hydrochloride was provided by SBI Pharmaceuticals Co., Ltd. SFC was obtained from Komatsuya Corporation. Dulbecco's modified Eagle's medium (DMEM), horse serum, HEPES, $\mathrm{NaCl}, \mathrm{KCl}, \mathrm{MgSO}_{4}, \mathrm{KH}_{2} \mathrm{PO}_{4}, \mathrm{CaCl}_{2}$, phloretin, Tris- $\mathrm{HCl}$ and dithiothreitol (DTT) were purchased from FUJIFILM Wako Pure Chemical Corporation.

Cell culture and treatment. C2C12 myotube cells (CRL-1772; American Type Culture Collection) were cultured at $37^{\circ} \mathrm{C}$ under $5 \% \mathrm{CO}_{2}$ using high-glucose DMEM (4,500 mg/l glucose) supplemented with $10 \%$ fetal bovine serum (Thermo Fisher Scientific, Inc.). For myotube differentiation, C2C12 cells were cultured for 5-7 days in high-glucose DMEM supplemented with $2 \%$ horse serum, and differentiated myotube cells were used for every experiment. For 2-deoxyglucose (2DG) uptake analysis, differentiated $\mathrm{C} 2 \mathrm{C} 12$ cells were incubated in low-glucose DMEM (1,000 mg/l glucose) containing 2\% horse serum for $18 \mathrm{~h}$ in a 12 -well plate. Subsequently, the cells were treated with or without 5-ALA/SFC in low-glucose DMEM containing $0.1 \%$ bovine serum albumin (BSA; Sigma-Aldrich; Merck KGaA) for $6 \mathrm{~h}$ at $37^{\circ} \mathrm{C}$. Doses of 5-ALA (100, 250, $500 \mu \mathrm{M})$ and $\mathrm{SFC}(50,125,250 \mu \mathrm{M})$ were determined from previous studies $(11,13,17)$. The cells were washed three times with $37^{\circ} \mathrm{C}$ Krebs-Ringer's phosphate (KRPH) buffer $(20 \mathrm{mM}$ HEPES, $137 \mathrm{mM} \mathrm{NaCl}, 4.7 \mathrm{mM} \mathrm{KCl}, 1 \mathrm{mM} \mathrm{MgSO}{ }_{4}, 5 \mathrm{mM}$ $\mathrm{KH}_{2} \mathrm{PO}_{4}, 1 \mathrm{mM} \mathrm{CaCl}$; $\mathrm{pH}$ 7.4) and incubated for $18 \mathrm{~min}$ at $37^{\circ} \mathrm{C}$ in $0.1 \%$ BSA-added KRPH buffer with or without insulin (Thermo Fisher Scientific, Inc.), 5-ALA, SFC or 5-ALA/SFC. The amount of 2DG uptake in $\mathrm{C} 2 \mathrm{C} 12$ cells was measured according to the manufacturer's protocol of the 2DG Uptake Measurement kit (Cosmo Bio Co., Ltd.). Next, 2DG was added to a final concentration of $1 \mathrm{mM}$, and incubation was continued for $20 \mathrm{~min}$ at $37^{\circ} \mathrm{C}$. This reaction was stopped by adding an ice-cold KRPH buffer containing $200 \mu \mathrm{M}$ phloretin (FUJIFILM Wako Pure Chemical Corporation). Subsequently, cells were washed three times with ice-cold KRPH buffer. Cells were sonicated for $30 \mathrm{sec}$ on ice with $10 \mathrm{mM}$ Tris- $\mathrm{HCl}$ (pH 8.0). The sonicated cellular solutions were heated at $85^{\circ} \mathrm{C}$ for $15 \mathrm{~min}$ and centrifuged at $16,000 \mathrm{x}$ g for $20 \mathrm{~min}$ at $4{ }^{\circ} \mathrm{C}$. Supernatants were used to measure $2 \mathrm{DG}$ amounts and protein concentrations. For western blotting analysis, differentiated $\mathrm{C} 2 \mathrm{C} 12$ cells were cultured in a $10-\mathrm{cm}$ dish.

Animals. The animal experiment protocols in the present study were approved by the Institutional Animal Care and Use Committee of Kobe University (approval no. 24-04-02; Kobe) and were conducted according to the guidelines set by this institution. A total of $18 \mathrm{C} 57 \mathrm{BL} / 6 \mathrm{~N}$ male mice (19-week-old; Japan SLC, Inc.) were acclimated at $23 \pm 3^{\circ} \mathrm{C}$ and relative humidity of $50 \pm 10 \%$ in a $12 \mathrm{~h}$ light-dark cycle (light from 8:00 a.m. to 8:00 p.m.), with water and a normal diet (ND) feed (cat. no. D12450B; Research Diets, Inc.) provided ad libitum for 1 week. After a week of ND feed, mice (body weight, 29-36 g) were categorized into three groups according to the oral administration for 4 weeks: i) ND Group with saline administration (ND; $n=5$ ); ii) HFD group with saline administration (HFD; 60 Kcal\% fat; cat. no. D12492; Research Diets, Inc.; n=8); and iii) HFD group with $300 \mathrm{mg} / \mathrm{kg} 5-\mathrm{ALA}$ and $47.1 \mathrm{mg} / \mathrm{kg}$ SFC administration (HFD + 5-ALA/SFC; $\mathrm{n}=5$ ). Body weights, food intake and water intake of mice were measured every day during the experiment.

On the final day, all mice were fasted for $5 \mathrm{~h}$. After fasting, mixtures of $2 \mathrm{~g} / \mathrm{kg}$ glucose and $3.2 \mathrm{mg} / \mathrm{kg} 2 \mathrm{DG}$ were intraperitoneally injected into ND, HFD and HFD + 5-ALA/SFC groups (each group, $n=5$ ) for intraperitoneal glucose tolerance test (IPGTT) and glucose uptake measurement. A total of three mice in the HFD group were intraperitoneally injected with glucose only as a negative control for 2DG. After measuring blood glucose levels, all mice were anesthetized with an intraperitoneal injection of $60 \mathrm{mg} / \mathrm{kg}$ pentobarbital and euthanized by exsanguination until cardiac arrest. Mortality was confirmed by respiratory arrest and reflex activity arrest. Organ samples were collected and stored at $-80^{\circ} \mathrm{C}$. Frozen samples were analyzed for 2DG uptake measurement and western blotting as described below.

IPGTT. IPGTT was performed after $5 \mathrm{~h}$ fasting (8:00 a.m. to 1:00 p.m.). Blood was collected from the tail veins of the mice. Blood glucose concentrations were measured at 0,15 , 30, 60, 90 and $120 \mathrm{~min}$ after the injection using Stat Strip XP (Nipro Corporation). The areas under the curve (AUCs) were calculated by the linear trapezoid method (18).

Measurement of 2DG uptake in mouse tissues. Amounts of 2DG uptake were measured according to the protocol of 2DG uptake measurement kit (Cosmo Bio Co., Ltd.) (19). Protein concentrations of the samples were determined using Pierce ${ }^{\circledR}$ $\mathrm{BCA}^{\mathrm{TM}}$ Protein Assay kit (Thermo Fisher Scientific, Inc.). Frozen mouse tissues were homogenized with an electric homogenizer at 10-40 times the weights of the dilution solution included in the kit. Homogenized solutions were heated at $85^{\circ} \mathrm{C}$ for $15 \mathrm{~min}$, followed by centrifugation at $16,000 \mathrm{x} \mathrm{g}$ for $20 \mathrm{~min}$ at $4^{\circ} \mathrm{C}$. Supernatants were used to measure $2 \mathrm{DG}$ uptake.

Western blotting. Extractions of the membrane and total proteins from $\mathrm{C} 2 \mathrm{C} 12$ cells were performed as previously described (20). Briefly, treated cells were homogenized in $50 \mathrm{mM}$ Tris- $\mathrm{HCl}$ (pH 8.0), containing $0.5 \mathrm{mM}$ DTT, $1 \%$ Halt 
Table I. Body and organ weights in mice fed with ND, HFD or HFD + 5-ALA/SFC administration for 4 weeks .

\begin{tabular}{lccc}
\hline Weights & ND & HFD & HFD + 5-ALA/SFC \\
\hline Body weight, g & $32.94 \pm 0.94$ & $40.38 \pm 0.95^{\mathrm{a}}$ & $36.76 \pm 0.54^{\mathrm{b}, \mathrm{c}}$ \\
Liver, g & $1.26 \pm 0.14$ & $1.26 \pm 0.09$ & $1.40 \pm 0.04$ \\
Spleen, mg & $71.74 \pm 7.91$ & $85.58 \pm 4.41$ & $67.02 \pm 4.68$ \\
Kidney, g & $0.31 \pm 0.01$ & $0.36 \pm 0.02$ & $0.34 \pm 0.01$ \\
Brown fat, g & $0.24 \pm 0.03$ & $0.40 \pm 0.08$ & $0.31 \pm 0.06$ \\
White fat, g & $4.37 \pm 0.40$ & $7.67 \pm 0.56^{\mathrm{d}}$ & $6.58 \pm 0.60^{\mathrm{b}}$ \\
Retroperitoneal and perirenal fat, g & $0.67 \pm 0.07$ & $1.11 \pm 0.08^{\mathrm{d}}$ & $0.87 \pm 0.08$ \\
Epididymal fat, g & $1.36 \pm 0.13$ & $2.22 \pm 0.19^{\mathrm{d}}$ & $1.80 \pm 0.14$ \\
Mesenteric fat, g & $0.54 \pm 0.04$ & $0.87 \pm 0.07^{\mathrm{d}}$ & $0.67 \pm 0.06$ \\
Subcutaneous fat, g & $1.81 \pm 0.20$ & $3.44 \pm 0.39^{\mathrm{b}}$ & $3.24 \pm 0.46^{\mathrm{b}}$ \\
\hline
\end{tabular}

Values are expressed as mean \pm SEM. $n=5-8$. ND, normal diet; HFD, high-fat diet; 5-ALA, 5-aminolevulinic acid; SFC, sodium ferrous citrate. ${ }^{\mathrm{a}} \mathrm{P}<0.001,{ }^{\mathrm{b}} \mathrm{P}<0.05$ vs. ND group; ${ }^{\mathrm{c}} \mathrm{P}<0.05$ vs. HFD group; ${ }^{\mathrm{d}} \mathrm{P}<0.01$ vs. ND group.

Protease Inhibitor Cocktail (Thermo Fisher Scientific, Inc.) and phosphatase inhibitors (Thermo Fisher Scientific, Inc.) (solution A). To prepare total cell lysate, a homogenized solution was gently stirred with an equal volume of solution $\mathrm{A}$ containing $1.0 \%$ NP-40, $0.5 \%$ sodium deoxycholate and $0.1 \%$ sodium dodecyl sulfate (SDS) on ice for $1 \mathrm{~h}$. To prepare the plasma membrane, another homogenized solution was centrifuged at 3,000 x $\mathrm{g}$ for $10 \mathrm{~min}$ at $4^{\circ} \mathrm{C}$. The washed precipitate was reacted for $1 \mathrm{~h}$ with gentle stirring on ice, then centrifuged at $20,000 \times \mathrm{g}$ for $20 \mathrm{~min}$ at $4^{\circ} \mathrm{C}$. The proteins $(5-20 \mu \mathrm{g} / \mathrm{lane})$ were subjected to SDS-PAGE using a 4-15\% Miniprotein ${ }^{\circledR}$ $\mathrm{TGX}^{\mathrm{TM}}$ precast gel (Bio-Rad Laboratories, Inc.) and transferred to a PVDF membrane (Bio-Rad Laboratories, Inc.). After blocking the membrane with 5\% non-fat milk in TBS with $0.05 \%$ Tween-20 (TBST) for $1 \mathrm{~h}$ at room temperature, the membrane was incubated with an anti-GLUT1 antibody (cat. no. 12939; Cell Signaling Technology, Inc.) and anti-insulin receptor $\beta$ (IR- $\beta$ ) antibody (cat. no. sc-711; Santa Cruz Biotechnology, Inc.) overnight at $4^{\circ} \mathrm{C}$. After washing, the membranes were incubated with HRP-linked donkey anti-rabbit IgG antibody (cat. no. NA934; Cytiva) for $1 \mathrm{~h}$ at room temperature, and developed with Immuno Star LD (cat. no. 292-699003; FUJIFILM Wako Pure Chemical Corporation) using ChemiDoc MP Imaging System (Bio-Rad Laboratories, Inc.). Image Lab software 4.0.1 (Bio-Rad Laboratories, Inc.) was used for densitometry. GLUT4 and GAPDH detections were conducted using the same membranes after stripping antibodies with Restore ${ }^{\mathrm{TM}}$ Western Blot Stripping Buffer (Thermo Fisher Scientific, Inc.). The stripped membrane was blocked with 5\% milk in TBST and reacted with anti-GLUT4 antibody (cat. no. 2213; Cell Signaling Technology, Inc.) or anti-GAPDH antibody (cat. no. E1C604-1; EnoGene Biotech Co, Ltd.) overnight at $4^{\circ} \mathrm{C}$. HRP-linked sheep anti-mouse IgG antibody (cat. no. NA931; Cytiva) for GLUT4 and HRP-linked donkey anti-rabbit IgG antibody (cat. no. NA934; Cytiva) for GAPDH were used. IR- $\beta$ was used as an internal control in the plasma membrane according to the previous study (21), and GAPDH was used as a control for the total protein. The data were analyzed using the ChemiDoc MP Imaging System (Bio-Rad Laboratories, Inc.).
Statistical analysis. Data were analyzed using GraphPad Prism 7 (GraphPad Software, Inc.). Results are expressed as means \pm standard error of the mean. In vivo data were statistically analyzed by one-way ANOVA with Tukey's test. In vitro data were statistically analyzed by one-way ANOVA with Dunnett's test. $\mathrm{P}<0.05$ was considered to indicate a statistically significant difference.

\section{Results}

5-ALA/SFC improves glucose tolerance in HFD-fed mice via upregulation of glucose uptake in muscle tissues. The effects of 5-ALA/SFC on body weight and glucose tolerance in HFD-fed mice were investigated. HFD-fed mice became $23 \%$ heavier compared with ND-fed mice, which was a significant change (Fig. 1A; Table I). No significant differences in food and water intakes were observed among the HFD and HFD + 5-ALA/SFC groups (data not shown). Body weights were significantly lighter in the HFD + 5-ALA/SFC group compared with the HFD group at 4 weeks after the administration (Fig. 1A; Table I). Total white fat weights were significantly heavier in the HFD group compared with the ND group (Table I). By contrast, white fat weights were not significant change between the HFD + 5-ALA/SFC and HFD groups (Table I). Next, the effect of 5-ALA/SFC on glucose tolerance was confirmed (Fig. 1B). Plasma glucose levels from 15-60 min after glucose administration were significantly higher in the HFD group compared with the ND group (Fig. 1B). Plasma glucose levels were significantly lower in the HFD + 5-ALA/SFC group compared with the HFD group at $30 \mathrm{~min}$ (Fig. 1B). Moreover, the AUC of plasma glucose levels was significantly lower in the HFD + 5-ALA/SFC group compared with the HFD group (Fig. 1C), suggesting that 5-ALA/SFC prevented obesity and improved glucose tolerance in HFD-fed mice.

Glucose uptakes in various tissues were investigated in the ND, HFD and HFD + 5-ALA/SFC groups by measuring the amount of 2DG uptake (Fig. 1D), a non-metabolized glucose analog (20). The average amount of 2DG uptake in gastrocnemius muscle was significantly lower in the HFD 

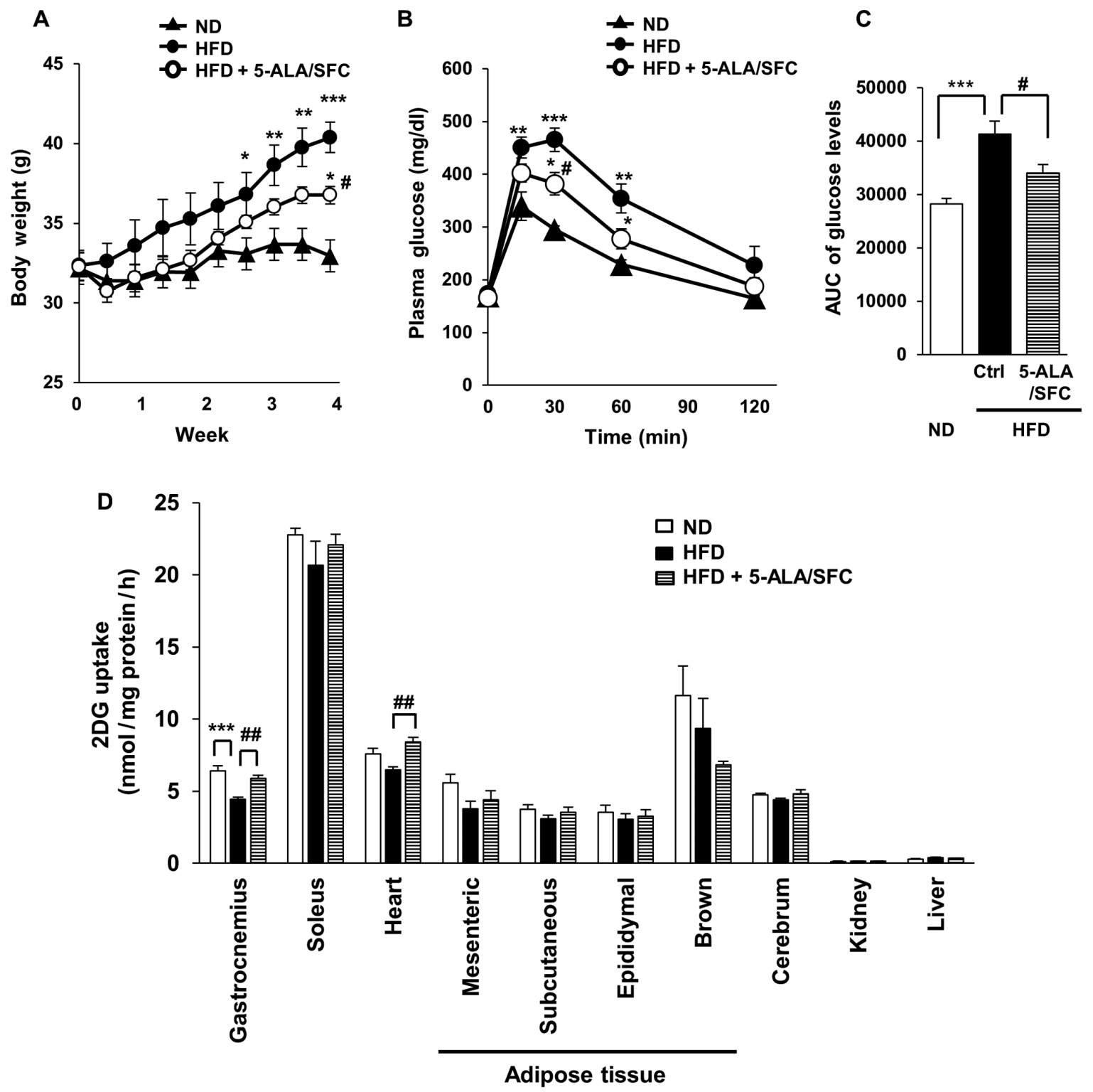

Figure 1. 5-ALA/SFC enhances glucose tolerance in the high-fat diet fed group by increasing glucose uptake in muscle tissues. (A) Body weight for 4 weeks after the administration of 5-ALA/SFC or saline. (B) Plasma glucose levels during IPGTT. (C) AUC of glucose levels during IPGTT. (D) 2DG uptake into each tissue in the ND, HFD and HFD + 5-ALA/SFC groups. $\mathrm{n}=5-8 .{ }^{*} \mathrm{P}<0.05,{ }^{* *} \mathrm{P}<0.01,{ }^{* * *} \mathrm{P}<0.01$ vs. ND group; ${ }^{\#} \mathrm{P}<0.05,{ }^{\# \#} \mathrm{P}<0.01$ vs. HFD group. AUC, area under the curve; IPGTT, intraperitoneal glucose tolerance test; 5-ALA, 5-aminolevulinic acid; SFC, sodium ferrous citrate; 2DG, 2-deoxyglucose; ND, normal diet, HFD, high-fat diet.

group compared with in ND group (Fig. 1D). The uptake of 2DG was slightly lower in soleus muscle, heart, white adipose tissues, brown adipose tissue and cerebrum in the HFD groups compared with those in the ND groups; however, the differences were not significant. By contrast, 2DG uptake levels in gastrocnemius muscle and heart were significantly higher in the HFD + 5-ALA/SFC group compared with those in the HFD group (Fig. 1D). The uptakes of 2DG in soleus muscle, white adipose tissues and cerebrum were markedly higher in the HFD + 5-ALA/SFC groups compared with the HFD groups, but these were not significant (Fig. 1D). Glucose uptake was upregulated by treatment with 5-ALA/SFC in gastrocnemius muscle and heart of HFD diabetic mice, followed by the improvement in glucose tolerance. Subsequently, the cellular mechanism underlying glucose uptake by 5-ALA/SFC was investigated using C2C12 myotube cells.
5-ALA/SFC upregulates GLUT1 translocation to the plasma membrane of C2C12 mouse myotube cells. The effect of 5-ALA/SFC on glucose uptake was investigated in $\mathrm{C} 2 \mathrm{C} 12$ mouse myotube cells. Differentiated C2C12 cells were used to investigate the mechanism underlying 2DG uptake by insulin stimulation. 2DG uptake was significantly increased by treatment with a combination of $>250 \mu \mathrm{M} 5$-ALA and $>125 \mu \mathrm{M}$ SFC in $\mathrm{C} 2 \mathrm{C} 12$ cells compared with that of untreated cells (Fig. 2A). Next, glucose uptake in $\mathrm{C} 2 \mathrm{C} 12$ cells treated with 5-ALA alone, SFC alone and their combination were examined. 5-ALA alone significantly increased 2DG uptake, but SFC alone did not change 2DG uptake in $\mathrm{C} 2 \mathrm{C} 12$ cells relative to that in the untreated control cells (Fig. 2B). The average amount of 2DG uptake with $250 \mu \mathrm{M}$ 5-ALA/125 $\mu \mathrm{M}$ SFC was a significant increase of $145 \%$ relative to that in untreated controls (Fig. 2C). This increase in 5-ALA/SFC 

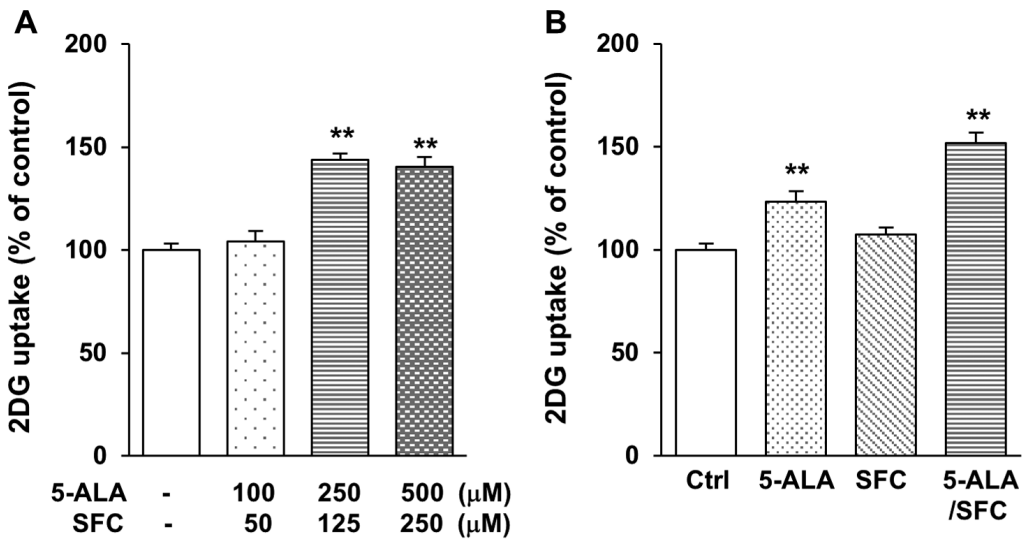

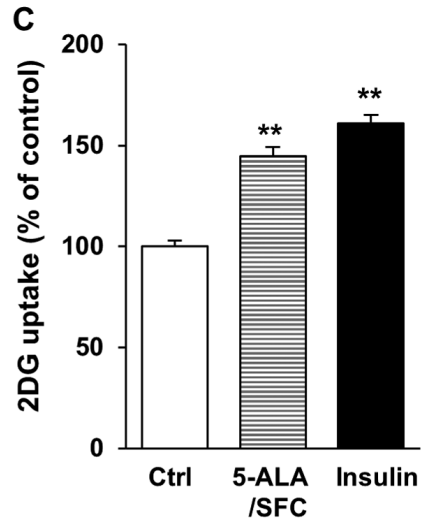

PM

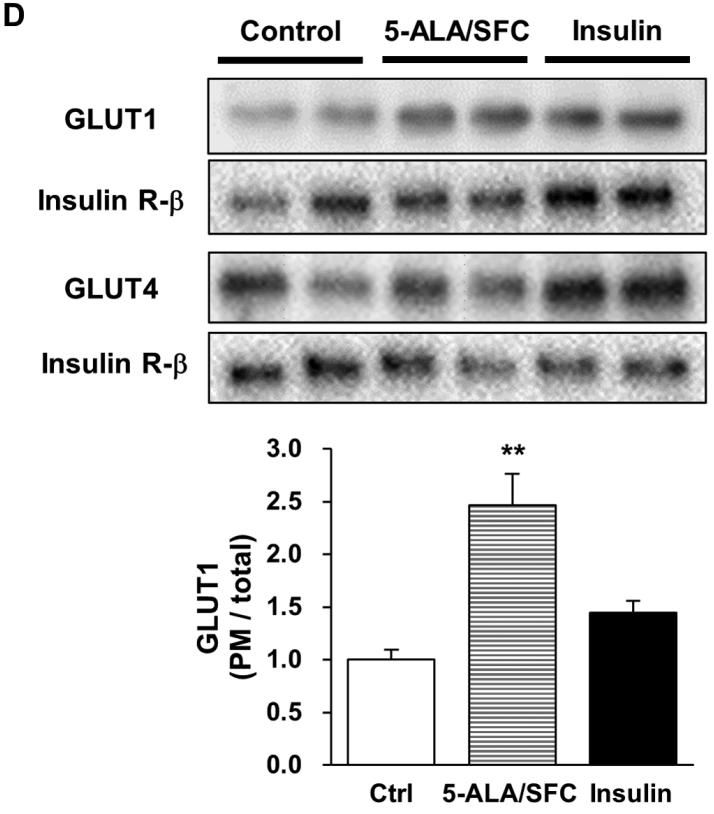

total
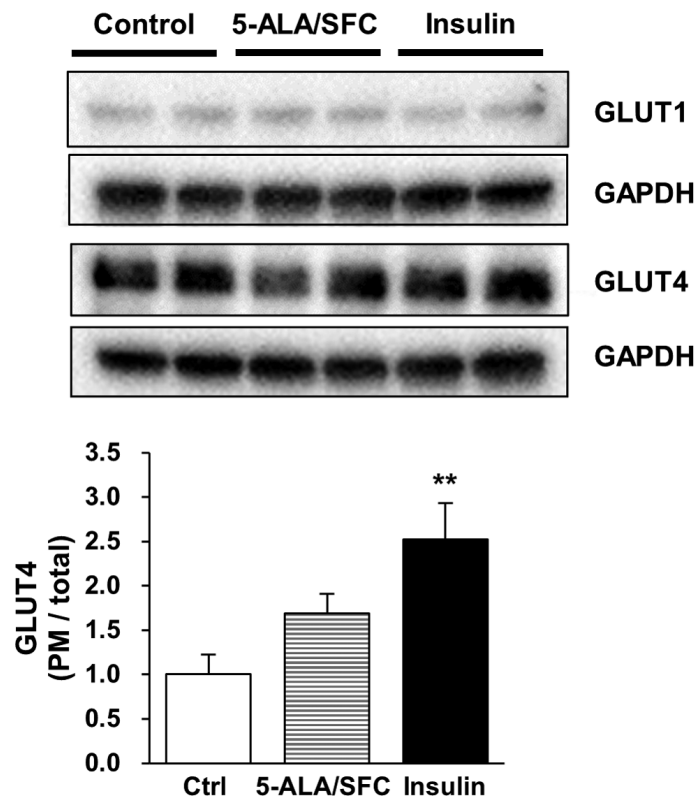

Figure 2. 5-ALA/SFC promotes glucose uptake and GLUT1 translocation in the plasma membrane of C2C12 mouse myotube cells. (A) 2DG uptake level is dependent on the concentration of treatment with 5-ALA/SFC. (B) 2DG uptake level for differences between 5-ALA alone, SFC alone and their combination. (C) Comparison of 2DG uptake level after treatment with 5-ALA/SFC or insulin (100 nM). (D) GLUT1 and GLUT4 protein expression levels in the plasma membrane relative to those in the total protein after treatment with 5-ALA/SFC or insulin. $\mathrm{n}=3$. ${ }^{* *} \mathrm{P}<0.01 \mathrm{vs}$. ctrl. Ctrl, control; 5-ALA, 5-aminolevulinic acid; SFC, sodium ferrous citrate; GLUT, glucose transporter; 2DG, 2-deoxyglucose; PM, plasma membrane.

treatment was equivalent to the increase observed in insulin treatment (Fig. 2C).

Next, GLUT1 and GLUT4 translocations in C2C12 cells were examined using western blotting. Sodium potassium ATPase 1a in the plasma membrane was decreased by 5-ALA/SFC (data not shown), thus IR- $\beta$ was used as a control in the plasma membrane in the present study. GLUT1 expression in the plasma membrane was significantly increased by 5 -ALA/SFC by 2.5 -fold relative to the control (Fig. 2D). Insulin markedly increased GLUT1 expression in the plasma membrane by 1.4-fold relative to the control, but the difference was not statistically significant (Fig. 2D). By contrast, insulin significantly increased GLUT4 expression in the plasma membrane by 2.5 -fold relative to the control (Fig. 2D). GLUT4 expression in the plasma membrane was increased in 5-ALA/SFC-treated cells by 1.7-fold relative to that in the control, but this change was not significant (Fig. 2D). Overall, these results suggested that 5-ALA/SFC upregulated glucose uptake via GLUT1 translocation in myotube cells.

\section{Discussion}

The present study investigated the mechanism underlying the 5-ALA/SFC-mediated increase in glucose uptake using HFD-fed diabetic mice in vivo and $\mathrm{C} 2 \mathrm{C} 12$ myotube cells in vitro. Administration of 5-ALA/SFC suppressed body weight gain and recovered impaired glucose tolerance in HFD-fed mice. These results are consistent with those of our previous report (13), which suggests that improved obesity and glucose tolerance are directly caused by 5 -ALA/SFC to reduce fat accumulation and promote glucose uptake from blood to cells. Additionally, this study revealed that 5-ALA/SFC enhances glucose uptake in 3T3-L1 adipocytes by 70-90\% and rat L6 myoblasts by $30 \%$ relative to the uptake in untreated cells (13). The present study confirmed that both 5-ALA alone and 5-ALA/SFC promoted glucose uptake in mouse $\mathrm{C} 2 \mathrm{C} 12$ myotube cells. Additionally, the target organs in mice with increased glucose uptake by 5-ALA/SFC were demonstrated to mainly be the gastrocnemius muscle and the heart. It was 
suggested that the 5-ALA/SFC-mediated increase of glucose uptake in gastrocnemius muscle and heart indicated the existence of an unknown mechanism by which 5-ALA/SFC enhances glucose uptake specifically in these muscles.

Muscle is a major contributor to the basal metabolic rate of the whole body $(22,23)$ and is responsible for $20 \%$ of the energy consumption in the whole body at rest $(23,24)$. Thus, glucose uptake in muscles can be more energy efficient compared with glucose uptake in other organs. The present study revealed that glucose uptake is the highest in soleus muscle among all examined organs; however, soleus muscle was not affected by 5-ALA/SFC. It was hypothesized that glucose uptake in both gastrocnemius muscle and heart sufficiently lowered systemic glucose concentrations in HFD-fed mice, because the weight of the soleus was lighter compared with the weight of the gastrocnemius. When calculated as the total amount of 2DG uptake per organ, gastrocnemius muscle demonstrated greater uptake compared with soleus muscle. Decreased blood glucose level in the HFD + 5-ALA/SFC group was considered to be the result of increased direct glucose uptake from blood to muscle tissues.

The current study demonstrated that the combination treatment of 5-ALA and SFC on C2C12 cells enhanced glucose uptake. SFC alone did not affect glucose uptake, whereas 5-ALA alone treatment weakly enhanced glucose uptake in $\mathrm{C} 2 \mathrm{C} 12$ cells. These results are consistent with our previous report on glucose uptake in rat L6 skeletal muscle cells (13), and also suggested the importance of 5-ALA metabolization to heme by adding SFC to increase glucose uptake in muscle tissues. Presumably, treatment with 5-ALA alone resulted in the metabolization of some 5-ALA to heme by using iron in a culture medium, which slightly increased the glucose uptake in $\mathrm{C} 2 \mathrm{C} 12$ cells.

5-ALA is considered a precursor of the mitochondrial electronic transport chain complex and upregulates ATP production by inducing the expression levels of complex IV and ATP synthase in vivo $(9,25)$. Overall, the combination of 5-ALA and SFC improves aerobic metabolism, thereby promoting cellular glucose consumption and leading to lower blood glucose levels $(11,13)$. Glucose uptake in muscle tissues is known to be caused mainly by insulin-independent GLUT1 and insulin-dependent GLUT4 (16,26-28). In the present study, the $\mathrm{C} 2 \mathrm{C} 12$ cell experiment results demonstrated an insignificant increase of GLUT1 translocation in insulin treatment compared with controls. This indicated that a significant induction of GLUT1 translocation may not always occur after insulin treatment. A previous report demonstrated that GLUT1 translocation is unchanged by insulin treatment in L6 cells (29). In the present study, 5-ALA/SFC significantly increased GLUT1 translocation from the cytoplasm to the plasma membrane compared with that of GLUT4 in C2C12 cells, without affecting GLUT1 expression. GLUT4 is required for acute insulin- and contraction-induced glucose uptake in skeletal muscle. However, the effect of GLUT1 on the skeletal muscle glucose uptake remains unclear. GLUT1 is widely expressed in various types of cells and tissues, such as erythrocytes and the brain $(30,31)$. GLUT1 is responsible for basal glucose transport and necessary for cell survival (30). Thus, the effect of 5-ALA/SFC on the membrane localization of GLUT1 may increase systemic glucose metabolism. Further examination of the 5-ALA/SFC mechanisms in diabetic mice or human muscle cells will be carried out in the future.
The presentstudy has some potentiallimitations.First,C2C12 cells are mouse myotube cells, thus the effect of 5-ALA/SFC on GLUT1 translocation in human cell lines has not been verified. The purpose of these cellular experiments was to clarify the contribution of GLUT as a result of 5-ALA/SFC-induced glucose uptake in HFD-fed mice. C2C12 cells are a well-documented model of diabetes (32). Differentiated C2C12 cells have myosin and glycogen, and closely mimic human myotube (32); therefore, this effect of 5-ALA/SFC was considered similar to humans. However, an additional study using a human cell line should be conducted in the future. Second, the present study has a lack of knockdown or inhibitor experiments; therefore, the mechanism of 5-ALA/SFC on GLUT1 translocation may not be accurately concluded from these results. STF31, the only GLUT-1 specific inhibitor, blocks GLUT1 activity by inhibiting NAD synthase (33-35). Thus, STF31 inhibits mitochondrial function. It is hypothesized that 5-ALA/SFC-induced glucose uptake results from enhanced mitochondrial function. STF31 would possibly antagonize the effect of 5-ALA/SFC by inhibiting mitochondrial function. Therefore, STF31 is considered inappropriate to evaluate the effectiveness of 5-ALA/SFC. On the other hand, GLUT1 siRNA is likely to affect cell survival and differentiation. Differentiated cells that express GLUT1 and GLUT4 are essential for the glucose uptake test (19), and mixed culture of un-differential cells cannot be evaluated. GLUT1 siRNA can affect the experimental system itself as well as GLUT1 interference. Resolving these limitations are still difficult to achieve and are a future challenge.

In conclusion, the current study demonstrated that 5-ALA/SFC enhanced glucose uptake in skeletal muscles and heart, and prevented impaired glucose tolerance in HFD-fed mice. Moreover, it was revealed that GLUT1 translocation may be involved in this mechanism. However, further studies are warranted to elucidate the molecular mechanisms underlying GLUT1 translocation by 5-ALA/SFC. The present results suggested that 5-ALA/SFC may be a useful drug for the treatment of diabetes, particularly in patients with insulin resistance and resistance to existing antidiabetic drugs.

\section{Acknowledgements}

The authors thank Dr Hidemitsu Sugihara and Dr Motowo Nakajima (SBI Pharmaceuticals Co., Ltd., Tokyo, Japan) for their critical reading of the manuscript and helpful discussion.

\section{Funding}

The present study was funded by SBI Pharmaceuticals Co., Ltd.

\section{Availability of data and materials}

The datasets generated and/or analyzed during the current study are not publicly available due to the commercial restrictions of SBI Pharmaceuticals Co., Ltd., but are available from the corresponding author on reasonable request.

\section{Authors' contributions}

YK, MI, YY and HA conceived and designed the experiments. YK and TI performed the experiments. YK, AK and 
TI confirm the authenticity of all the raw data and interpreted the data. YK and AK statistically analyzed the data and wrote the manuscript. All authors have read and approved the final manuscript.

\section{Ethics approval and consent to participate}

The animal experiments in the present study were approved by the Institutional Animal Care and Use Committee of Kobe University (approval no. 24-04-02; Kobe, Japan).

\section{Patient consent for publication}

Not applicable.

\section{Competing interests}

YK, AK, TI and MI are employees of SBI Pharmaceuticals Co., Ltd. YY and HA declare that they have no competing interests.

\section{References}

1. Hendry GA and Jones OT: Haems and chlorophylls: Comparison of function and formation. J Med Genet 17: 1-14, 1980.

2. Castro CE: Mechanisms of reaction of hemeproteins with oxygen and hydrogen peroxide in the oxidation of organic substrates. Pharmacol Ther 10: 171-189, 1980.

3. Ishizuka M, Abe F, Sano Y, Takahashi K, Inoue K, Nakajima M, Kohda T, Komatsu N, Ogura S and Tanaka T: Novel development of 5-aminolevurinic acid (ALA) in cancer diagnoses and therapy. Int Immunopharmacol 11: 358-365, 2011.

4. Stummer W, Pichlmeier U, Meinel T, Wiestler OD, Zanella F and Reulen HJ; ALA-Glioma Study Group: Fluorescence-guided surgery with 5-aminolevulinic acid for resection of malignant glioma: A randomised controlled multicentre phase III trial. Lancet Oncol 7: 392-401, 2006.

5. Inoue K, Fukuhara H, Shimamoto T, Kamada M, Iiyama T, Miyamura M, Kurabayashi A, Furihata M, Tanimura M, Watanabe $\mathrm{H}$, et al: Comparison between intravesical and oral administration of 5-aminolevulinic acid in the clinical benefit of photodynamic diagnosis for nonmuscle invasive bladder cancer. Cancer 118: 1062-1074, 2012.

6. Antonini $\mathrm{E}$ and Brunori M: Hemoglobin and myoglobin in their reactions with ligands. In: Frontiers of biology. North-Holland Publication Co., Amsterdam, p436, 1971.

7. Sono M,Roach MP,CoulterED and Dawson JH:Heme-Containing Oxygenases. Chem Rev 96: 2841-2888, 1996.

8. Paterniti JR Jr, Lin CI and Beattie DS: delta-Aminolevulinic acid synthetase: Regulation of activity in various tissues of the aging rat. Arch Biochem Biophys 191: 792-797, 1978.

9. Ogura S, Maruyama K, Hagiya Y, Sugiyama Y, Tsuchiya K, Takahashi K, Abe F, Tabata K, Okura I, Nakajima M, et al: The effect of 5-aminolevulinic acid on cytochrome c oxidase activity in mouse liver. BMC Res Notes 4: 66, 2011.

10. Masuki S, Morita A, Kamijo Y, Ikegawa S, Kataoka Y, Ogawa Y, Sumiyoshi E, Takahashi K, Tanaka T, Nakajima M, et al: Impact of 5-aminolevulinic acid with iron supplementation on exercise efficiency and home-based walking training achievement in older women. J Appl Physiol (1985) 120: 87-96, 2016.

11. Hara T, Koda A, Nozawa N, Ota U, Kondo H, Nakagawa H, Kamiya A, Miyashita K, Itoh H, Nakajima M, et al: Combination of 5-aminolevulinic acid and ferrous ion reduces plasma glucose and hemoglobin A1c levels in Zucker diabetic fatty rats. FEBS Open Bio 6: 515-528, 2016

12. Higashikawa F, Noda M, Awaya T, Tanaka T and Sugiyama M: 5-aminolevulinic acid, a precursor of heme, reduces both fasting and postprandial glucose levels in mildly hyperglycemic subjects. Nutrition 29: 1030-1036, 2013.

13. Ota U, Hara T, Nakagawa $\mathrm{H}$, Tsuru E, Tsuda M, Kamiya A, Kuroda Y,Kitajima Y, Koda A, Ishizuka M, et al: 5-aminolevulinic acid combined with ferrous ion reduces adiposity and improves glucose tolerance in diet-induced obese mice via enhancing mitochondrial function. BMC Pharmacol Toxicol 18: 7, 2017.
14. Klip A, McGraw TE and James DE: Thirty sweet years of GLUT4. J Biol Chem 294: 11369-11381, 2019.

15. Gallagher D, Belmonte D, Deurenberg P, Wang Z, Krasnow N, Pi-Sunyer FX and Heymsfield SB: Organ-tissue mass measurement allows modeling of REE and metabolically active tissue mass. Am J Physiol 275: E249-E258, 1998.

16. Wood IS and Trayhurn P: Glucose transporters (GLUT and SGLT): Expanded families of sugar transport proteins. Br J Nutr 89: 3-9, 2003

17. Chibazakura T, Toriyabe Y, Fujii H, Takahashi K, Kawakami M, Kuwamura H, Haga H, Ogura S, Abe F, Nakajima M, et al: 5-Aminolevulinic acid enhances cell death under thermal stress in certain cancer cell lines. Biosci Biotechnol Biochem 79: 422-431, 2015.

18. Allison DB, Paultre F, Maggio C, Mezzitis N and Pi-Sunyer FX: The use of areas under curves in diabetes research. Diabetes Care 18: 245-250, 1995.

19. Saito K, Lee S, Shiuchi T, Toda C, Kamijo M, Inagaki-Ohara K, Okamoto S and Minokoshi Y: An enzymatic photometric assay for 2-deoxyglucose uptake in insulin-responsive tissues and 3T3-L1 adipocytes. Anal Biochem 412: 9-17, 2011.

20. Suzuki K and Kono T: Evidence that insulin causes translocation of glucose transport activity to the plasma membrane from an intracellular storage site. Proc Natl Acad Sci USA 77: 2542-2545, 1980.

21. Jiang H, Yamashita Y, Nakamura A, Croft K and Ashida H: Quercetin and its metabolite isorhamnetin promote glucose uptake through different signalling pathways in myotubes. Sci Rep 9: 2690, 2019.

22. Kinney JM and Tucker HN: Energy Metabolism. Tissue Determinants and Cellular Corollaries. Ravan Press, New York, NY, p562, 1992.

23. Zurlo F, Larson K, Bogardus C and Ravussin E: Skeletal muscle metabolism is a major determinant of resting energy expenditure. J Clin Invest 86: 1423-1427, 1990.

24. Durnin JV: Basal metabolic rate in man. In: Joint FAO/-WHO/ UNU Expert Consultation on Energy and Protein Requirements, 1981.

25. Fujii C, Miyashita K, Mitsuishi M, Sato M, Fujii K, Inoue H, Hagiwara A, Endo S, Uto A, Ryuzaki M, et al: Treatment of sarcopenia and glucose intolerance through mitochondrial activation by 5-aminolevulinic acid. Sci Rep 7: 4013, 2017.

26. Mueckler M: Family of glucose-transporter genes. Implications for glucose homeostasis and diabetes. Diabetes 39: 6-11, 1990.

27. Douen AG, Ramlal T, Rastogi S, Bilan PJ, Cartee GD, Vranic M, Holloszy JO and Klip A: Exercise induces recruitment of the 'insulin-responsive glucose transporter'. Evidence for distinct intracellular insulin- and exercise-recruitable transporter pools in skeletal muscle. J Biol Chem 265: 13427-13430, 1990.

28. Kraegen EW, Sowden JA, Halstead MB, Clark PW, Rodnick KJ, Chisholm DJ and James DE: Glucose transporters and in vivo glucose uptake in skeletal and cardiac muscle: Fasting, insulin stimulation and immunoisolation studies of GLUT1 and GLUT4. Biochem J 295: 287-293, 1993.

29. Yamashita Y, Okabe M, Natsume $M$ and Ashida $\mathrm{H}$ : Cacao liquor procyanidin extract improves glucose tolerance by enhancing GLUT4 translocation and glucose uptake in skeletal muscle. J Nutr Sci 1: e2, 2012.

30. Gould GW and Holman GD: The glucose transporter family: Structure, function and tissue-specific expression. Biochem J 295: 329-341, 1993.

31. Montel-Hagen A, Sitbon M and Taylor N: Erythroid glucose transporters. Curr Opin Hematol 16: 165-172, 2009.

32. Wong CY, Al-Salami H and Dass CR: C2C12 cell model: Its role in understanding of insulin resistance at the molecular level and pharmaceutical development at the preclinical stage. J Pharm Pharmacol 72: 1667-1693, 2020.

33. Chan DA, Sutphin PD, Nguyen P, Turcotte S, Lai EW, Banh A, Reynolds GE, Chi JT, Wu J, Solow-Cordero DE, et al: Targeting GLUT1 and the Warburg effect in renal cell carcinoma by chemical synthetic lethality. Sci Transl Med 3: 94ra70, 2011.

34. Adams DJ, Ito D, Rees MG, Seashore-Ludlow B, Puyang X, Ramos AH, Cheah JH, Clemons PA, Warmuth M, Zhu P, et al: NAMPT is the cellular target of STF-31-like small-molecule probes. ACS Chem Biol 9: 2247-2254, 2014.

35. Garten A, Petzold S, Körner A, Imai S and Kiess W: Nampt: Linking NAD biology, metabolism and cancer. Trends Endocrinol Metab 20: 130-138, 2009. 